\title{
Effects of aging on cardiac extracellular matrix in men and women
}

\author{
Elke Dworatzek ${ }^{1,2}$, Istvan Baczko ${ }^{3}$ and Georgios Kararigas ${ }^{1,2}$ \\ ${ }^{1}$ Institute of Gender in Medicine and Center for Cardiovascular Research, Charite University Hospital, Berlin, \\ Germany \\ 2 DZHK (German Centre for Cardiovascular Research), Berlin Partner Site, Berlin, Germany \\ ${ }^{3}$ Department of Pharmacology and Pharmacotherapy, University of Szeged, Szeged, Hungary
}

Purpose: Aging has severe implications for tissue damage and is a major risk factor for disease. However, the effects of aging on cardiac extracellular matrix (ECM) components in individuals free of cardiovascular disease are incompletely understood. We aimed at the characterization of the effects of aging on major ECM proteins in the heart of men and women.

Experimental design: Left ventricular (LV) samples of nondiseased human hearts technically unusable for transplantation obtained from general organ donors $(n=31$; age 17-68 years; $48 \%$ women) were used for protein isolation. We separated the group into $17-40$ years $(n=7$ men and 7 women) and 50-68 years ( $n=9$ men and 8 women).

Results: Analysis of ECM proteins demonstrated an age-dependent sex-specific regulation of collagen type I and III (interaction $p<0.05$ ), type VI (interaction $p=0.01$ ), tissue inhibitor of metalloproteinase 3 (interaction $p<0.05$ ), SMAD2 (interaction $p<0.05$ ), and SMAD3 (interaction $p=0.001$ ). Overall, the levels of these proteins in younger individuals were lower in women than men, while in older individuals they were higher in women than men.

Conclusions and clinical relevance: This age-mediated myocardial ECM remodeling might play a key role in the limited ability of the aging heart to adapt adequately to altered work load and to respond to tissue damage. Therapeutic agents that target ECM homeostasis represent promising prevention strategies.

\section{Keywords:}

Aging / Extracellular matrix / Heart

\section{Introduction}

Aging is one of the major risk factors for disease. It favors tissue damage, while it hinders tissue repair. Homeostatic imbalance is an important hallmark of aging. In the heart, aging is characterized by a progressive alteration of myocardial structure and is a risk factor for cardiac morbidity and mortality [1]. Age-dependent myocardial remodeling is, among others, related with changes in the amount and organization of extracellular matrix (ECM) components [2].

Correspondence: Dr. Georgios Kararigas, Institute of Gender in Medicine and Center for Cardiovascular Research, Charite University Hospital, Hessische Str. 3-4, 10115 Berlin, Germany

E-mail: george.kararigas@charite.de

Fax: $+49-30-450525943$

Abbreviations: ECM, extracellular matrix; LV, left ventricular; MMP, matrix metalloproteinase; SMAD, SMAD family member; TGF $\beta$, transforming growth factor $\beta$; TIMP, tissue inhibitor of metalloproteinase
Received: April 1, 2015

Revised: July 20, 2015

Accepted: August 3, 2015
In fact, the process of aging in the heart is associated with a loss of cardiomyocytes and subsequent hypertrophy of the remaining cells, which, in turn, may lead to alterations in ECM composition.

The myocardial ECM is mainly composed of fibrillar collagens, i.e. types I and III. Collagen degradation is mediated by matrix metalloproteinases (MMP). MMP activity is, in turn, inhibited by tissue-specific inhibitors of metalloproteinases (TIMP) [3]. An important factor for the maintenance of ECM integrity and stability is the transforming growth factor (TGF) $\beta$. In fact, activation of the TGF $\beta$ signaling pathway may induce factors with potent fibrogenic actions, such as periostin. The downstream effectors of TGF $\beta$ are the SMAD family member proteins, which, when phosphorylated, translocate to the nucleus, and activate gene transcription [4]. Structural proteins of the ECM, such as vimentin and vinculin, play a primary role in tissue architecture providing structural support and tissue organization. Furthermore,

Colour Online: See the article online to view Fig. 6 in colour. 


\section{Clinical Relevance}

Aging is a physiological process that also affects the heart with striking impact on clinical outcomes in healthy elderly individuals and in patients with cardiovascular disease. The interaction between ageinduced functional and structural changes and the actual mechanisms of disease will define the prognosis and severity of cardiovascular disease occurrence in aging populations. Myocardial remodeling due to alterations in extracellular matrix (ECM) components leads to cardiac dysfunction representing a major clinical burden. However, the role of aging in this process in men and women is not completely understood. The present study provides evidence of changes in ECM that may increase vulnerability of the human heart to tissue damage, thereby underlying the onset of age-related cardiac disease. Further knowledge on age-dependent alterations of the ECM will give rise to novel perspectives with diagnostic and therapeutic potential. Subsequently, the development of strategies targeting these factors is of clinical relevance.
ECM components mediate transduction of cell survival signals promoting tissue repair pathways, thereby maintaining function [5].

Aberrant induction of ECM components can initiate a cascade of events, including the activation of signal transduction pathways, which, in turn, activate cellular responses that may promote tissue damage [6]. The balance of ECM synthesis and degradation is essential for normal cardiac structure and function. The disruption of ECM homeostasis is a key factor for the progression of cardiac dysfunction [7]. Thus, a better understanding of the modulation of ECM regulatory components with aging is necessary for gaining novel insight into managing age-related cardiac tissue damage and dysfunction.

To this extent, significant age-related changes in myocardial ECM components, particularly higher left ventricular (LV) collagen content, have been reported in aged rats compared to young rats [8-10], mice [11-16], and in sheep [17]. However, how aging affects the myocardial ECM in humans is not fully understood. In the present study, we aimed at the characterization of the effects of aging on major ECM proteins in the human heart hypothesizing differences between men and women.

\section{Materials and methods}

\subsection{Collection of left ventricular tissues}

Samples from the lateral LV wall of nondiseased human hearts technically unusable for transplantation obtained from general organ donors ( $n=31 ; 48 \%$ women) were frozen immediately in liquid nitrogen until further processing. In particular, we divided our samples into two age groups, i.e. 1740 years ( $n=7$ men and 7 women) and 50-68 years $(n=9$ men and 8 women). We excluded the age group $40-50$ years to avoid the peri-menopausal phase of female individuals with highly irregular levels of sex hormones, particularly estrogens. Experimental protocols were approved by the Scientific and Research Ethical Committee of the Medical Scientific Board at the Hungarian Ministry of Health (ETT-TUKEB:
4991-0/2010-1018EKU), followed the principles outlined in the Declaration of Helsinki and written consent was obtained from all donors.

\subsection{Protein extraction and immunoblotting}

LV samples were homogenized in a modified RIPA buffer (50 mM Tris- $\mathrm{HCl}, \mathrm{pH}$ 7.4, $150 \mathrm{mM} \mathrm{NaCl}, 1 \mathrm{mM}$ EDTA, $1 \%$ NP-40, 0.25\% Na-deoxycholate) supplemented with protease inhibitor cocktail (Roche, Mannheim, Germany) and the phosphatase inhibitors sodium orthovanadate $(1 \mathrm{mM}$ Na3VO4) and sodium fluoride (1 $\mathrm{mM} \mathrm{NaF})$. Proteins were quantified using the BCA Assay (Thermo Scientific Pierce Protein Biology, Schwerte, Germany), resolved by SDSPAGE, transferred to nitrocellulose membranes using standard procedures. Membranes were probed with primary antibodies: collagen 1A2 (M-80, Santa Cruz, Dallas, TX, USA), collagen 3A1 (H-300, Santa Cruz, Dallas, TX, USA), collagen 6A1 (H-200, Santa Cruz, Dallas, TX, USA), MMP2 (Cell Signaling, Danvers, MA, USA), MMP9 (abcam, Cambridge, UK), TIMP1 (H-150, Santa Cruz, Dallas, TX, USA), TIMP3 (H-55, Santa Cruz, Dallas, TX, USA), periostin (S-15, Santa Cruz, Dallas, TX, USA), TGFß1 (Santa Cruz, Dallas, TX, USA), SMAD2 (Cell Signaling, Danvers, MA, USA), SMAD3 (Cell Signaling, Danvers, MA, USA), p-SMAD2 (Cell Signaling, Danvers, MA, USA), p-SMAD3 (Cell Signaling, Danvers, MA, USA), SMAD4 (Cell Signaling, Danvers, MA, USA), vimentin (Sigma, Germany), vinculin (Sigma, Germany), and HSP60 (Enzo, USA) serving as loading control. Immunoreactive proteins were detected using ECL Plus (GE Healthcare, Buckinghamshire, UK) and quantified by the Image 1.41 version software (http://rsbweb.nih.gov/ij/).

\subsection{Histology}

Frozen LV tissue samples were cut into $5 \mu \mathrm{m}$ sections and stained with picro-sirius red staining for collagen content using standard procedures as described previously $[18,19]$. 


\subsection{Statistical analysis}

Data are shown as mean \pm SEM. Comparisons among multiple groups were performed using 2-way ANOVA, which assesses the effect of two different categorical independent variables on one continuous dependent variable. This statistical test assesses the main effect of each independent variable alone, as well as if there is any interaction between these two independent variables. In other words, with 2-way ANOVA the effects of aging and sex alone, as well as the interaction of both factors on ECM protein abundance were assessed. Tukey's post-hoc test was used to adjust for multiple pairwise comparisons. All tests were performed with the $\mathrm{R}$ version 2.14.2 software considering $p \leq 0.05$ significant.

\section{Results}

\subsection{Study design}

To study the effects of aging on major ECM proteins of the human myocardium, we collected LV samples of male and female donors $(n=31)$ with no apparent cardiovascular disease. We separated the group into younger (17-40 years; $n=$ 7 male and 7 female) and older (50-68 years; $n=9$ male and 8 female) individuals. Therefore, the present study design offered the possibility to perform an integrative investigation of the effects of aging, sex, and the interaction of both factors on ECM protein abundance. We focused our efforts on proteins with a known and major role in ECM homeostasis, remodeling, and the regulation thereof, such as collagens, MMPs and TIMPs. We also assessed proteins of the TGF $\beta$ signaling pathway, which is an important player in the pathogenesis of cardiac remodeling and fibrosis, as well as two proteins necessary for cytoskeletal integrity and structural adaptation.

\subsection{Collagens}

Collagens are the main component of myocardial ECM, with collagen types I and III being the most abundant ECM proteins, and are essential for cardiac structure and function. The levels of collagen type I (COL1A2) and III (COL3A1) were modulated by aging in a sex-specific manner (interaction $p<0.05$; Fig. 1). In younger individuals, women had less collagen types I and III than men, while in older individuals, women had more collagen types I and III than men. In addition, aging alone led to significantly increased levels of collagen type III in older women compared to younger women (adjusted $p<0.05$; Fig. 1B).

Similarly, the levels of collagen type VI (COL6A1) were modulated by aging in a sex-specific manner (interaction $p=$ 0.01 ; Fig. 1B). In younger individuals, women had less collagen type VI than men, while in older individuals, women had more collagen type VI than men. In this case, the difference between younger men and younger women showed a statistical trend (adjusted $p<0.1$ ).
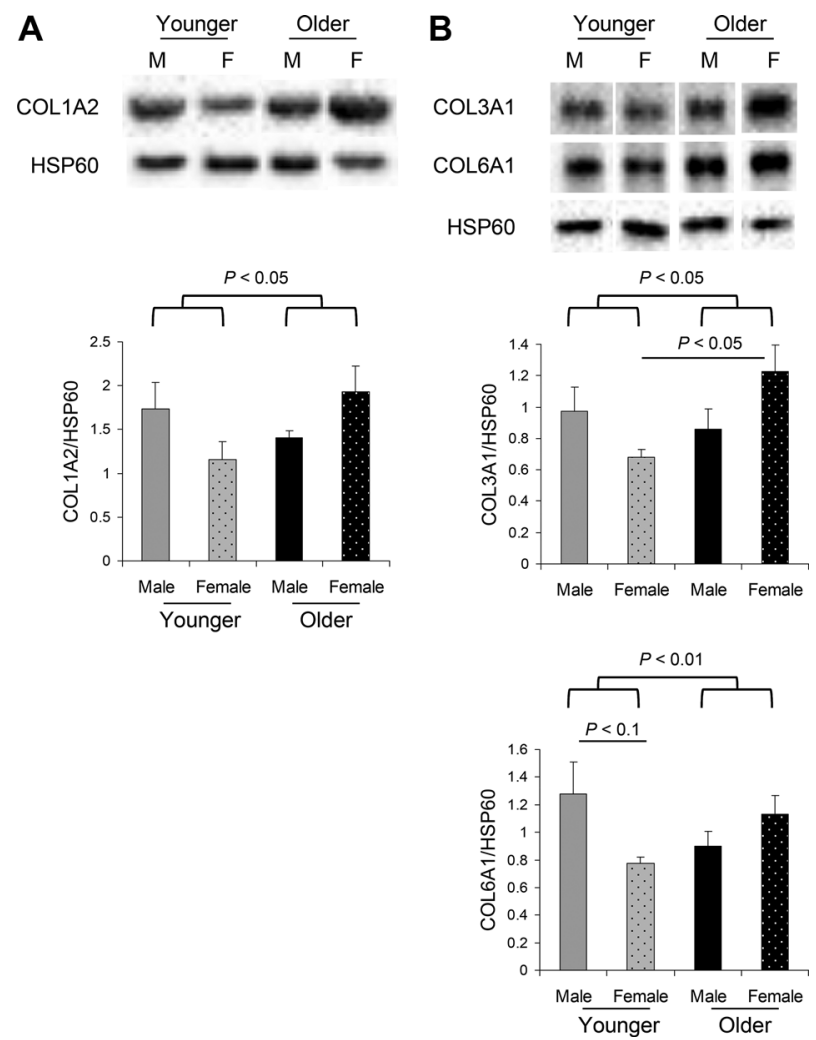

Figure 1. Effects of aging, sex, or the interaction of both factors on collagens. Collagen type I (COL1A2) and III (COL3A1) were modulated by aging in a sex-specific manner (interaction $p<$ 0.05). Similarly, collagen type VI (COL6A1) levels were modulated by aging in a sex-specific manner (interaction $p=0.01$ ). Older women had significantly increased levels of collagen type III compared with younger women (adjusted $p<0.05$ ). In all figures: representative images of immunoblotting analysis with antibodies specific for the indicated proteins in left ventricular tissue samples from younger (17-40 years) and older (50-68 years) men and women are shown; the lanes were run on the same gel but were noncontiguous; HSP60 served as loading control; semiquantification of protein profiling by means of densitometry analysis is shown; data present mean \pm SEM; the top $p$ value is used to indicate the effect of the interaction of aging and sex; $n=7$ younger male; $n=7$ younger female; $n=9$ older male; $n=8$ older female.

\subsection{Matrix metalloproteinases}

MMPs initiate and degrade collagens and are involved in the decomposition of collagen degradation products. The levels of MMP2 and MMP9 were not significantly changed (Fig. 2A and B). However, a statistical trend was found in the levels of MMP9 due to the interaction between aging and sex (interaction $p<0.1$; Fig. 2B).

\subsection{Tissue inhibitor of metalloproteinases}

TIMPs are the major inhibitors of MMPs and therefore involved in ECM remodeling. The levels of TIMP3 were 


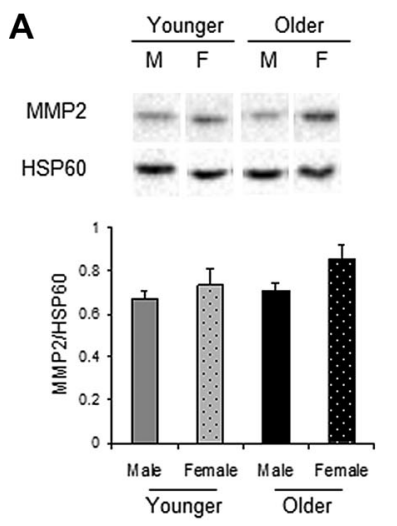

B

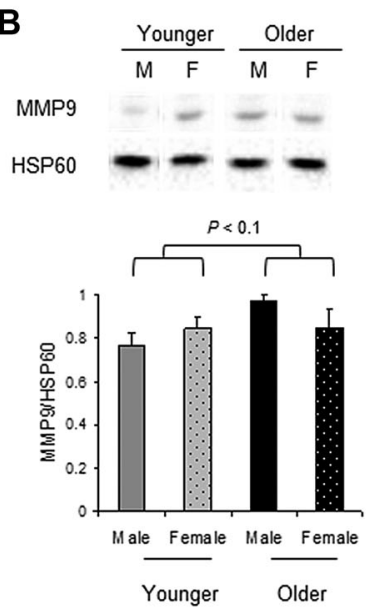

On the other hand, the levels of TIMP2 were significantly higher in older women than in older men (adjusted $p<$ 0.05 ), younger women (adjusted $p<0.05$ ), and younger men (adjusted $p=0.01$; Fig. 2D).

\subsection{Transforming growth factor $\beta$ signaling proteins}

TGF $\beta$ and its downstream effectors the SMAD proteins are involved in cardiac remodeling by regulating ECM gene expression. The levels of TGF $\beta$ were significantly lower in younger individuals than in older individuals $(p=0.05$; Fig. 3A). There was no further statistically significant effect or trend on this protein.

The levels of SMAD2 were modulated by aging in a sexspecific manner (interaction $p<0.05$; Fig. 3B). In younger individuals, women had less SMAD2 than men, while in older individuals, women had more SMAD2 than men. Notably, older women had significantly more SMAD2 than older men (adjusted $p=0.01$ ) and younger women (adjusted $p=0.05$; Fig. 3B). However, there was no statistical significance or trend for the levels of phosphorylated SMAD2 related to total SMAD2.

Similarly, the levels of SMAD3 were also modulated by aging in a sex-specific manner (interaction $p=0.001$; Fig. $3 \mathrm{C}$ ). In younger individuals, women had less SMAD3 than men, while in older individuals, women had more SMAD3 than men. Notably, older women had significantly more SMAD3 than older men (adjusted $p<0.05$ ) and younger women (adjusted $p<0.05$; Fig. 3C). Furthermore, the levels of phosphorylated SMAD3 related to total SMAD3 were significantly higher in older women than in older men (adjusted $p<0.05$; Fig. 3C).

Significant effects on the levels of SMAD4 were found due to aging $(p<0.001)$ and sex $(p=0.01)$, and the interaction of both factors had a strong statistical trend $(p=0.06)$. Notably, older women had significantly more SMAD4 than older men (adjusted $p<0.05$ ), younger men (adjusted $p<0.001$ ), and younger women (adjusted $p<0.01$; Fig. 3D).

Figure 2. Effects of aging, sex, or the interaction of both factors on matrix metalloproteinases and tissue inhibitor of metalloproteinases. There was no significant change of MMP2 (A) and MMP9 (B) proteins. TIMP3 (C) was modulated by aging in a sex-specific manner (interaction $p<0.05$ ). There was no significant change of TIMP1 (C). TIMP2 (D) levels were significantly higher in older women than in older men (adjusted $p<0.05$ ), younger women (adjusted $p<0.05$ ), and younger men (adjusted $p=0.01$ ).

modulated by aging in a sex-specific manner (interaction $p<0.05$; Fig. 2C). In younger individuals, women had less TIMP3 than men, while in older individuals, women had more TIMP3 than men.

A similar regulation was found for TIMP1. However, the interaction between aging and sex showed a statistical trend (interaction $p<0.1$; Fig. 2C).

\subsection{Cytoskeletal proteins}

Vimentin and vinculin are important proteins of the cytoskeleton necessary for the maintenance of cardiac structure and function. The levels of vimentin were significantly higher in younger individuals than in older individuals $(p<0.001$; Fig. 4A). In addition, there was a significant effect of the interaction between aging and sex (interaction $p=0.01$ ). In younger individuals, women had more vimentin than men, while in older individuals, women had less vimentin than men. Notably, older women had significantly less vimentin than older men (adjusted $p=0.05$ ) and younger women (adjusted $p<0.001$; Fig. 4A).

The levels of vinculin showed a statistical trend due to the interaction between aging and sex (interaction $p<0.1$; 
A
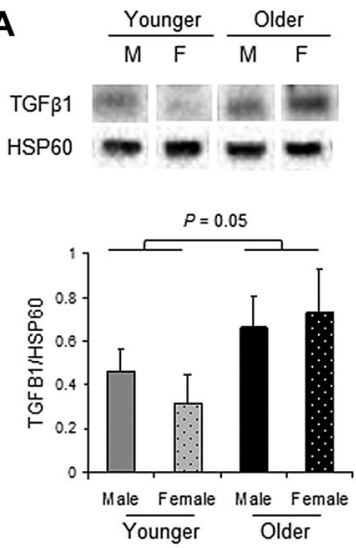

B
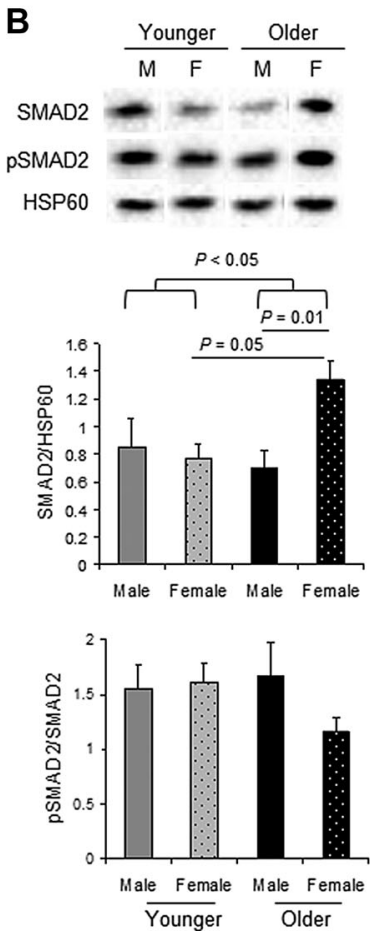
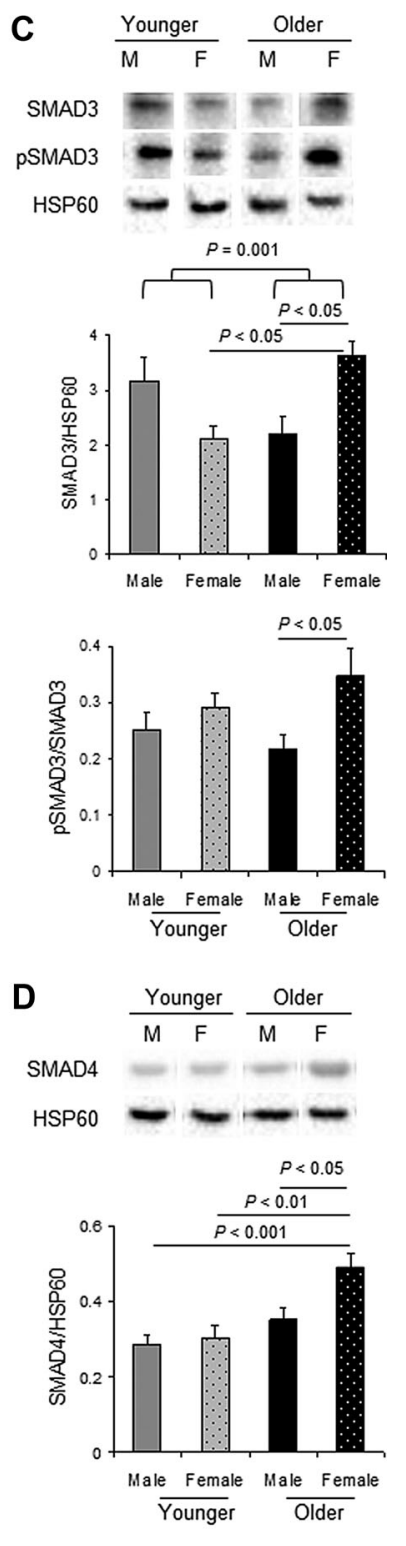

A
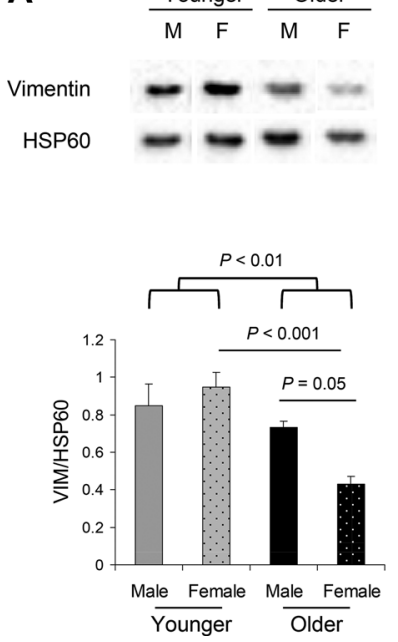

B

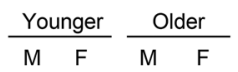

Vinculin
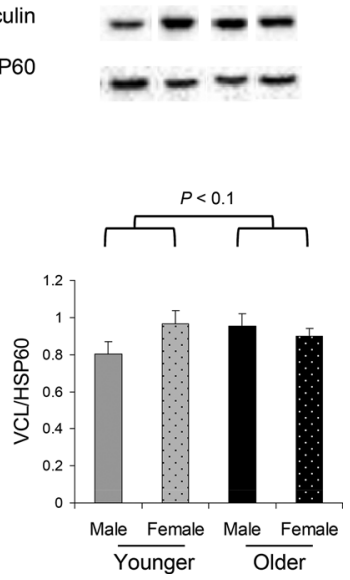

Figure 4. Effects of aging, sex, or the interaction of both factors on vimentin (A) and vinculin (B). Vimentin was modulated by aging in a sex-specific manner (interaction $p=0.01$ ). Additionally, vimentin levels were significantly higher in younger individuals compared with older individuals $(p<0.001)$. Notably, older women had significantly less vimentin than older men (adjusted $p=0.05$ ) and younger women (adjusted $p<0.001$ ). On the other hand, vinculin protein levels were not significantly changed.

Fig. 4B). This trend revealed that in younger individuals, women had more vinculin than men, while in older individuals, women had less vinculin than men.

\subsection{Matricellular protein periostin}

Periostin is a matricellular protein associated with cardiac collagen deposition exerting potent fibrogenic actions. There was no statistically significant effect or trend on the levels of periostin (Fig. 5).

\subsection{Histological analysis of collagen content}

To further assess the effects of aging on ECM homeostasis in the human heart, we performed picro-sirius red staining to quantify morphometric analysis of collagen content in histological sections. There was no statistically significant effect or trend on collagen content (Fig. 6).

\section{Discussion}

In the current study, we present data on the effects of aging on major ECM components in hearts of men and women. Our findings provide insight into potential age-dependent mechanisms that might contribute to increased vulnerability of the human heart to tissue damage. In particular, we found that the protein levels of collagen types I, III, and VI, 


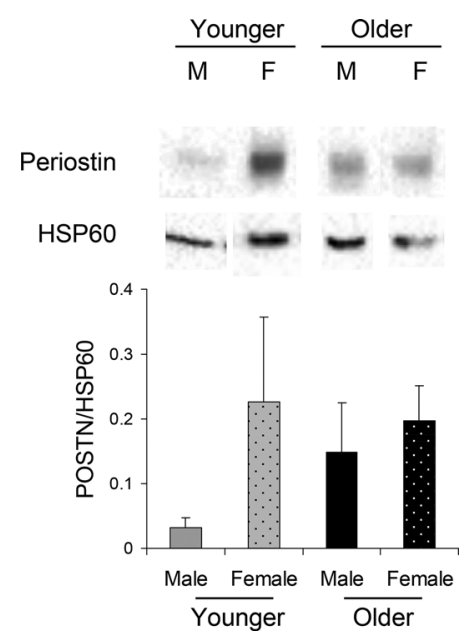

Figure 5. Effects of aging, sex, or the interaction of both factors on periostin. Periostin protein levels were not significantly changed in left ventricular tissue samples from younger and older men and women.
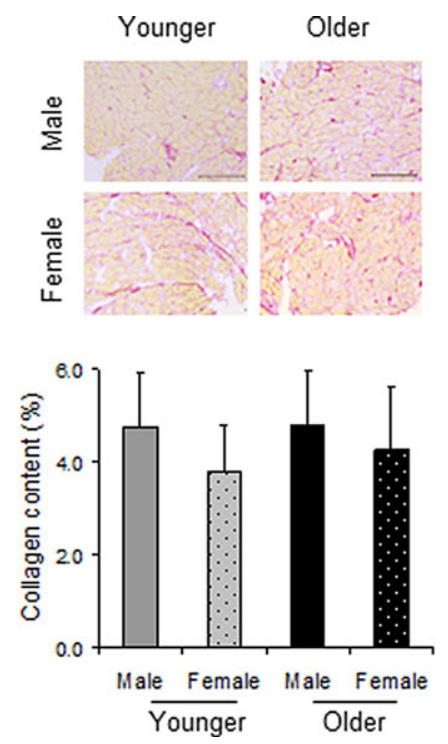

Figure 6. Effects of aging, sex, or the interaction of both factors on collagen content assessed histologically. Representative picro-sirius red staining images and quantification of collagen content in histological sections of left ventricular samples from younger and older men and women. There was no significant change of the heart collagen content detected. Data present mean \pm SEM. Scale bar: $200 \mu \mathrm{m} ; n=5$ /group.

TIMP3, SMAD2, and SMAD3 were lower in women than men in younger individuals, while in older individuals they were higher in women than men.

Several studies employing rodents have shown significant increases in myocardial ECM components with aging [812]. Furthermore, a study combining electron microscopy and histochemical analysis of human hearts revealed that collagen types I and III increase in number and thickness as a function of age [20]. However, the effects of aging on myocardial ECM protein levels in men and women free of cardiovascular disease are not fully understood. To this extent, we found a generalized tendency toward an induction of ECM components in the hearts of older women.

The myocardium is susceptible to tissue damage triggered by several factors, such as ischemic injury, excessive inflammation, and aberrantly altered work load. Due to limited cardiac tissue regenerative capacity, tissue repair is mainly mediated through the modulation of the myocardial ECM, especially collagens [21]. As a result, collagen synthesis and degradation dysregulation favors interstitial and perivascular collagen deposition, leading to increased myocardial stiffness and cardiac dysfunction [22]. Consequently, during cardiac senescence, induction of ECM proteins may play a role in the maintenance of the structural integrity of the heart, but on the other hand it may be involved in the pathogenesis of age-related fibrosis.

In particular, excessive deposition and cross-linking of ECM components in the myocardium play an important role in the pathogenesis of diastolic heart failure, i.e. heart failure with preserved ejection fraction, in aging hearts [23, 24], and may lead to arrhythmias [25]. This age-related increase of fibrillar collagen content and myocardial diastolic stiffness appears to be dependent on mechanisms regulating the processing of newly synthesized procollagen into mature collagen fibrils [13]. Notably, exercise training was shown to protect the aging heart against dysregulation of MMPs and fibrosis by suppressing elevation of TIMP1 and TGF $\beta$ [26]. Women suffer more commonly from diastolic heart failure than men [27]. Women are also more susceptible to drug-induced QT prolongation, torsades de pointes, and paroxysmal supraventricular tachycardia [28-31]. Therefore, the coordinated increase in collagen and TIMP protein levels we identified in the aging female myocardium might contribute to the higher incidence of diastolic dysfunction in women and might predispose them to arrhythmias.

The mechanisms responsible for the age-dependent increases in myocardial ECM components are unclear. ECM homeostasis and collagen content in the myocardium could be due to the regulation of collagen and other factors at the transcriptional level. It is likely that the regulatory elements involved in this process may include steroid hormones, as some of the genes coding for these proteins have putative full or half-site steroid response elements in their sequences. Nevertheless, they have even been shown to be under the regulation of steroid hormones without the involvement of steroid response elements [32-34]. In fact, aging has been associated with changes in sex steroid hormones, particularly $17 \beta$-estradiol (E2), increased cardiac tissue damage and risk for cardiovascular disease. In previous studies, we have found that E2 exerts direct effects in the heart that maybe sex specific [35-40]. Notably, treatment of fibroblasts with E2 led to increased gene expression of collagen types I and III in males but to decreased levels of these genes in females [34]. Furthermore, E2 led to decreased MMP2 gene levels [32]. These 
findings indicate that the presence of E2 is necessary for the repression of ECM components in the female heart. On the basis of this, considering that the older women included in this study are most likely postmenopausal, the decreased levels of circulatory E2 might contribute to the increases in myocardial ECM components in this group.

In addition, it has to be noted that these increasing levels are not of the same degree as we have found previously for ECM and particularly fibrosis markers under diseased conditions, where male hearts show significantly higher levels than female hearts [41,42]. Besides, periostin, a mediator of fibrosis $[43,44]$, did not show any statistical regulation or trend also indicating that the age-related increase in ECM components in women is not of pathological nature. The SMAD proteins are the downstream effectors of TGF $\beta$. When SMAD2 and SMAD3 are phosphorylated, they bind to SMAD4 and translocate to the nucleus, where they activate gene transcription of profibrotic factors. In fact, it has been shown that the pSMAD3-SMAD4 complex mediates early ECM component regulation, while the pSMAD2-SMAD4 complex is responsible for long-term/persistent regulation processes [45]. Therefore, the higher levels of pSMAD3 and SMAD4 in older women compared to age-matched men in the absence of any differences in pSMAD2 levels also indicate a nonpathological regulation of myocardial ECM components in older women. Collectively, these findings indicate that aging alone in the female heart did not lead to the conversion of fibroblasts to myofibroblasts.

Our study was constrained by a relatively small sample size stratified by sex and high heterogeneity related to human biological material. Due to this, statistical significance might have not been reached in cases of a pronounced trend for sex differences. Furthermore, information on endogenous circulating hormones or exogenous administration of hormones was missing. We have also not established a connection between ECM and fibroblast numbers. This is certainly an exciting point for further investigation under appropriate experimental approaches, such as measurements of isolated fibroblasts from fresh cardiac tissues.

In summary, our study shows how aging affects major ECM proteins in the heart of men and women. The agedependent alterations in myocardial ECM components are expected to favor ECM accumulation and may contribute to mechanisms increasing the vulnerability of the heart to tissue damage.

We thank J. Thomas and V. Riese for technical assistance. We acknowledge support from the Hungarian Scientific Research Fund (OTKA K109610).

The authors have declared no conflict of interest.

\section{References}

[1] Lakatta, E. G., Levy, D., Arterial and cardiac aging: major shareholders in cardiovascular disease enterprises: part II: the aging heart in health: links to heart disease. Circulation 2003, 107, 346-354.

[2] Swynghedauw, B., Molecular mechanisms of myocardial remodeling. Physiol. Rev. 1999, 79, 215-262.

[3] Chakraborti, S., Mandal, M., Das, S., Mandal, A., Chakraborti, T., Regulation of matrix metalloproteinases: an overview. Mol. Cell. Biochem. 2003, 253, 269-285.

[4] Shi, Y., Massague, J., Mechanisms of TGF-beta signaling from cell membrane to the nucleus. Cell 2003, 113, 685-700.

[5] Rienks, M., Papageorgiou, A. P., Frangogiannis, N. G., Heymans, S., Myocardial extracellular matrix: an everchanging and diverse entity. Circ. Res. 2014, 114, 872-888.

[6] Hynes, R. O., The extracellular matrix: not just pretty fibrils. Science 2009, 326, 1216-1219.

[7] Baudino, T. A., Carver, W., Giles, W., Borg, T. K., Cardiac fibroblasts: friend or foe? Am. J. Physiol. Heart Circ. Physiol. 2006, 291, H1015-H1026.

[8] Lieber, S. C., Qiu, H., Chen, L., Shen, Y. T. et al., Cardiac dysfunction in aging conscious rats: altered cardiac cytoskeletal proteins as a potential mechanism. Am. J. Physiol. Heart Circ. Physiol. 2008, 295, H860-H866.

[9] Eghbali, M., Robinson, T. F., Seifter, S., Blumenfeld, O. O., Collagen accumulation in heart ventricles as a function of growth and aging. Cardiovasc. Res. 1989, 23, 723-729.

[10] Nguyen, C. T., Hall, C. S., Scott, M. J., Zhu, Q. et al., Age-related alterations of cardiac tissue microstructure and material properties in Fischer 344 rats. Ultrasound Med. Biol. 2001, 27, 611-619.

[11] Burgess, M. L., McCrea, J. C., Hedrick, H. L., Age-associated changes in cardiac matrix and integrins. Mech. Ageing Dev. 2001, 122, 1739-1756.

[12] Lindsey, M. L., Goshorn, D. K., Squires, C. E., Escobar, G. P. et al., Age-dependent changes in myocardial matrix metalloproteinase/tissue inhibitor of metalloproteinase profiles and fibroblast function. Cardiovasc. Res. 2005, 66, 410-419.

[13] Bradshaw, A. D., Baicu, C. F., Rentz, T. J., Van Laer, A. O. et al., Age-dependent alterations in fibrillar collagen content and myocardial diastolic function: role of SPARC in postsynthetic procollagen processing. Am. J. Physiol. Heart Circ. Physiol. 2010, 298, H614-H622.

[14] Dai, D. F., Santana, L. F., Vermulst, M., Tomazela, D. M. et al., Overexpression of catalase targeted to mitochondria attenuates murine cardiac aging. Circulation 2009, 119, 2789-2797.

[15] de Castro Bras, L. E., Toba, H., Baicu, C. F., Zile, M. R. et al., Age and SPARC change the extracellular matrix composition of the left ventricle. BioMed. Res. Int. 2014, 2014, 810562.

[16] Stein, M., Noorman, M., van Veen, T. A., Herold, E. et al., Dominant arrhythmia vulnerability of the right ventricle in senescent mice. Heart Rhythm 2008, 5, 438-448.

[17] Horn, M. A., Graham, H. K., Richards, M. A., Clarke, J. D. et al., Age-related divergent remodeling of the cardiac extracellular matrix in heart failure: collagen accumulation in the young and loss in the aged. J. Mol. Cell Cardiol. 2012, $53,82-90$.

[18] Dworatzek, E., Mahmoodzadeh, S., Schubert, C., Westphal, C. et al., Sex differences in exercise-induced physiological 
myocardial hypertrophy are modulated by oestrogen receptor beta. Cardiovasc. Res. 2014, 102, 418-428.

[19] Petrov, G., Dworatzek, E., Schulze, T. M., Dandel, M. et al., Maladaptive remodeling is associated with impaired survival in women but not in men after aortic valve replacement. JACC Cardiovasc. Imaging 2014, 7, 1073-1080.

[20] Mendes, A. B., Ferro, M., Rodrigues, B., Souza, M. R. et al., Quantification of left ventricular myocardial collagen system in children, young adults, and the elderly. Medicina 2012, 72, 216-220.

[21] Krieg, T., Le Roy, E. C., Diseases of the extracellular matrix. J. Mol. Med. 1998, 76, 224-225.

[22] Kong, P., Christia, P., Frangogiannis, N. G., The pathogenesis of cardiac fibrosis. Cell. Mol. Life Sci. 2014, 71, 549-574.

[23] Frangogiannis, N. G., Matricellular proteins in cardiac adaptation and disease. Physiol. Rev. 2012, 92, 635-688.

[24] Martos, R., Baugh, J., Ledwidge, M., O'Loughlin, C. et al., Diastolic heart failure: evidence of increased myocardial collagen turnover linked to diastolic dysfunction. Circulation 2007, 115, 888-895.

[25] de Jong, S., van Veen, T. A., van Rijen, H. V., de Bakker, J. M., Fibrosis and cardiac arrhythmias. J. Cardiovasc. Pharmacol. 2011, 57, 630-638.

[26] Kwak, H. B., Kim, J. H., Joshi, K., Yeh, A. et al., Exercise training reduces fibrosis and matrix metalloproteinase dysregulation in the aging rat heart. FASEB J. 2011, 25, 1106-1117.

[27] Cleland, J. G., Swedberg, K., Follath, F., Komajda, M. et al., The EuroHeart failure survey programme-a survey on the quality of care among patients with heart failure in Europe. Part 1: patient characteristics and diagnosis. Eur. Heart $J$. 2003, 24, 442-463.

[28] Drici, M. D., Knollmann, B. C., Wang, W. X., Woosley, R. L., Cardiac actions of erythromycin: influence of female sex. JAMA 1998, 280, 1774-1776.

[29] Lehmann, M. H., Hardy, S., Archibald, D., MacNeil, D. J., JTc prolongation with d,l-sotalol in women versus men. Am. J. Cardiol. 1999, 83, 354-359.

[30] Reinoehl, J., Frankovich, D., Machado, C., Kawasaki, R. et al., Probucol-associated tachyarrhythmic events and QT prolongation: importance of gender. Am. Heart J. 1996, 131, 11841191.

[31] Rodriguez, L. M., de Chillou, C., Schlapfer, J., Metzger, J. et al., Age at onset and gender of patients with different types of supraventricular tachycardias. Am. J. Cardiol. 1992, 70, 1213-1215.

[32] Mahmoodzadeh, S., Dworatzek, E., Fritschka, S., Pham, T. H., Regitz-Zagrosek, V., 17beta-Estradiol inhibits matrix metalloproteinase-2 transcription via MAP kinase in fibroblasts. Cardiovasc. Res. 2010, 85, 719-728.

[33] Mahmoodzadeh, S., Pham, T. H., Kuehne, A., Fielitz, B. et al., 17beta-Estradiol-induced interaction of ERalpha with NPPA regulates gene expression in cardiomyocytes. Cardiovasc. Res. 2012, 96, 411-421.

[34] Petrov, G., Regitz-Zagrosek, V., Lehmkuhl, E., Krabatsch, T. et al., Regression of myocardial hypertrophy after aortic valve replacement: faster in women? Circulation 2010, 122, S23-S28.

[35] Kararigas, G., Becher, E., Mahmoodzadeh, S., Knosalla, C. et al., Sex-specific modification of progesterone receptor expression by 17 beta-oestradiol in human cardiac tissues. Biol. Sex Differ. 2010, 1, 2

[36] Kararigas, G., Bito, V., Tinel, H., Becher, E. et al., Transcriptome characterization of estrogen-treated human myocardium identifies Myosin regulatory light chain interacting protein as a sex-specific element influencing contractile function. J. Am. Coll. Cardiol. 2012, 59, 410-417.

[37] Kararigas, G., Nguyen, B. T., Jarry, H., Estrogen modulates cardiac growth through an estrogen receptor alphadependent mechanism in healthy ovariectomized mice. Mol. Cell. Endocrinol. 2014, 382, 909-914.

[38] Kararigas, G., Nguyen, B. T., Zelarayan, L. C., Hassenpflug, M. et al., Genetic background defines the regulation of postnatal cardiac growth by 17 beta-estradiol through a beta-catenin mechanism. Endocrinology 2014, 155, 2667-2676.

[39] Kararigas, G., Fliegner, D., Forler, S., Klein, O. et al., Comparative proteomic analysis reveals sex and estrogen receptor beta effects in the pressure overloaded heart. J. Proteome Res. 2014, 13, 5829-5836.

[40] Kararigas, G., Fliegner, D., Gustafsson, J. A., RegitzZagrosek, V., Role of the estrogen/estrogen-receptor-beta axis in the genomic response to pressure overloadinduced hypertrophy. Physiol. Genomics 2011, 43, 438446.

[41] Kararigas, G., Dworatzek, E., Petrov, G., Summer, H. et al., Sex-dependent regulation of fibrosis and inflammation in human left ventricular remodelling under pressure overload. Eur. J. Heart Fail 2014, 16, 1160-1167.

[42] Petrov, G., Dworatzek, E., Schulze, T., Dandel, M. et al., Maladaptive remodeling is associated with impaired survival in women but not in men after aortic valve replacement. JACC Cardiovasc. Imaging 2014, 7, 1073-1080.

[43] Norris, R. A., Damon, B., Mironov, V., Kasyanov, V. et al., Periostin regulates collagen fibrillogenesis and the biomechanical properties of connective tissues. J. Cell. Biochem. 2007, 101, 695-711.

[44] Oka, T., Xu, J., Kaiser, R. A., Melendez, J. et al., Genetic manipulation of periostin expression reveals a role in cardiac hypertrophy and ventricular remodeling. Circ. Res. 2007, 101, 313-321.

[45] Yang, Y. C., Piek, E., Zavadil, J., Liang, D. et al., Hierarchical model of gene regulation by transforming growth factor beta. Proc. Natl. Acad. Sci. USA 2003, 100, 10269-10274. 